\title{
The Collaborative Crystallography program at the Advanced Light Source
}

Banumathi Sankaran, Corie Ralston, Jay Nix, James Holton, Scott Classen, Paul Adams

Lawrence Berkeley National Lab

The collaborative crystallography (CC) program at the Advanced Light Source is part of the NIH funded ALS-Enable program, which provides a single, integrated access mode for all X-ray related structural biology needs. The collaborative crystallography program provides basic crystallography services, ranging from crystallization to structure deposition and all steps in between. We will review and highlight past structures and present new opportunities the ALS-Enable program provides. 